\title{
Stimm- und Kehlkopfveränderungen im Alter (Presbyphonie und Presbylarynx)
}

\author{
Vocal Changes and Laryngeal Modifications in the Elderly \\ (Presbyphonia and Presbylarynx)
}

Autor

Institut
W. Angerstein

Selbstständiger Funktionsbereich für Phoniatrie und Pädaudiologie, Universitäts-Klinikum Düsseldorf
Schlüsselwörter

- Presbyphonie

- Presbylarynx

- Therapieoptionen

Keywords

- presbyphonia

- presbylarynx

therapeutic options

Bibliografie

DOI http://dx.doi.org/ 10.1055/s-0042-109595

Sprache · Stimme · Gehör 2016; 40: 131-135

C Georg Thieme Verlag KG

Stuttgart · New York

ISSN 0342-0477

Korrespondenzadresse

Univ.-Prof. Dr. med. Wolfgang Angerstein

Selbstständiger Funktionsbereich für Phoniatrie und

Pädaudiologie

Universitäts-Klinikum

Düsseldorf

Moorenstraße 5/Geb. 13.77

40225 Düsseldorf

angerstein@med.uni-

duesseldorf.de

\section{Zusammenfassung}

$\nabla$

Etwa 20\% der über 60-Jährigen weisen eine $\mathrm{Al}$ tersstimmstörung (Presbyphonie) auf, die oftmals von Presbyakusis (Altersschwerhörigkeit), Presbyphagie (Schluckstörungen im Alter), Presbyvertigo (Altersschwindel) und/oder Presbyopie (Alterssichtigkeit) begleitet wird.

Presbyphonie betrifft die Singstimme häufiger, früher und deutlicher als die Sprechstimme. Aber nicht nur Qualität und Belastbarkeit von Singund Sprechstimme werden beeinträchtigt, auch der Atemantrieb wird durch die eingeschränkte Lungenfunktion schwächer. Durch das Absenken des Kehlkopfes verändern sich bei Senioren zudem die Resonanzverhältnisse im Ansatzrohr, und das Aspirationsrisiko steigt.

Die stimmlichen Einbußen beruhen auf morphologischen Altersveränderungen des Kehlkopfes (Prebylarynx), die sowohl das ektolaryngeale Kehlkopfskelett als auch die endolaryngealen Weichteile (insbesondere die Stimmlippen) betreffen. Der Vokalismuskel atrophiert und wird hypoton, was zu einer spindelförmig-ovalären Glottisschlussinsuffizienz mit Luftverlust bei Phonation führt. Die Schleimhäute trocknen aus im Sinne einer Laryngitis sicca.

Bei der Stimmrehabilitation im Alter müssen allgemeine und speziell auf die Stimme bezogene Behandlungsoptionen unterschieden werden: $\mathrm{Zu}$ den allgemeinen Therapieansätzen gehört die Mitbehandlung internistischer und neurologischer Grunderkrankungen (z.B. Reflux, neurodegenerative oder zerebrovaskuläre Erkrankungen) - auch Nebenwirkungen von Medikamenten müssen beachtet werden. Ein gesunder Lebensstil ohne Noxen begünstigt den Funktionserhalt von Phonations- und Atemorganen. Stimmtherapeutisch kommen sowohl Tonisierungsübungen zur Verbesserung des phonatorischen Glottisschlusses als auch detonisierende Entspannungsübungen zum Abbau supraglotti-

\section{Abstract \\ $\nabla$}

In about $20 \%$ of the population older than 60 years of age, voice problems (presbyphonia) are manifest, often combined with hearing deficits (presbyacusis), swallowing disorders (presbyphagia), dizziness (presbyvertigo) and/or impaired vision (presbyopia).

The singing voice is afflicted more often, earlier and more severely than the speaking voice. But it is not just the vocal quality and capacity that are affected. Due to reduced pulmonary function, the breathing impetus is also weakened. As a result of the descending of the larynx, resonance properties of the vocal tract change in seniors, and there is increased risk of aspiration.

Vocal deficits are caused by age-related morphological changes in the larynx (presbylarynx). These alterations concern both the ectolaryngeal skeleton and the endolaryngeal soft tissues (particularly the vocal folds). The vocalis muscle atrophies and becomes hypotonic, which becomes clinically apparent as vocal fold bowing and spindle-shaped glottic chink with loss of air during phonation. The laryngeal mucosa dries out (laryngitis sicca).

Vocal rehabilitation in the elderly includes both general and voice-specific therapeutic options. Underlying internal and neurological diseases (e.g. reflux, neurodegenerative or cerebrovascular affections), as well as side effects of drugs must be considered. A healthy lifestyle without pathogenic agents promotes good function of phonatory and respiratory organs. As far as voice therapy is concerned, glottal attack exercises can improve glottal closure during phonation, and relaxation exercises may reduce supraglottic hyperfunctional compression. In special cases with large glottic chink, phonosurgical augmentation of the vocal folds may enhance glottal closure 
scher hyperfunktioneller Pressmechanismen in Betracht. In Einzelfällen mit großem Glottisspalt bei Phonation kann eine phonochirurgische Stimmlippenaugmentation den Glottisschluss verbessern und somit die Stimme kräftigen. Stimmhygienische Maßnahmen (z.B. regelmäßiges Befeuchten der Atemwege) ergänzen das Stimmtraining.

Singen im Alter hat sowohl psychosozial (Steigerung des Wohlbefindens und der sozialen Teilhabe) als auch immunologisch (Anstieg des IgA im Speichel) positive Effekte. and vocal strength. Vocal hygiene (e.g. regular airway moisturisation) complements voice exercises.

In the elderly, singing has proven pychosocial benefits (improvement of well-being and social participation) and verified positive immunological effects (salivary IgA increase).

\section{Lernziel}

Die klinische Symptomatik der Altersstimme sowie die zugrunde liegenden morphologischen Kehlkopfveränderungen werden vorgestellt, Behandlungsansätze der Altersstimme werden diskutiert.

\section{Einleitung \\ $\nabla$}

Prävalenz

Wir alle wissen, dass unsere Lebenserwartung aufgrund der demografischen Entwicklung ständig steigt. Die Menschen werden immer älter. Eine längere Lebenserwartung bedeutet jedoch auch eine längere Beanspruchung des Stimmapparates mit der Gefahr einer Stimmüberlastung insbesondere im fortgeschrittenen Lebensalter. Daher ist die demografische Entwicklung unserer modernen Kommunikationsgesellschaft mit dem Risiko einer Zunahme von Stimmstörungen im Alter (Presbyphonie) belastet. Die Häufigkeitsangaben über Stimmstörungen im Alter differieren zwischen 10 und 47\% [1-5], sodass zusammengefasst etwa $20 \%$ der über 60 -Jährigen eine Altersstimmstörung aufweisen dürften.

\section{Komorbiditäten}

Presbyphonie wird oftmals begleitet von Presbyakusis (Altersschwerhörigkeit [1]), Presbyphagie (Schluckstörungen im Alter [5]), Presbyvertigo (Altersschwindel) und/oder Presbyopie (Alterssichtigkeit). Die meisten gesicherten Daten betreffen die Presbyakusis: Etwa 50\% aller stimmgestörten älteren Patienten haben einen Hörverlust, und 10,5\% der Senioren haben zusätzlich zum Hörverlust Stimmprobleme [2]. Bei etwa 30\% der über 65-Jährigen ist die Höreinbuße so gravierend, dass eine Hörhilfe angezeigt ist [6]. Hörstörungen im Alter verschlechtern Qualität und Belastbarkeit der Stimme [7].

Die oben genannten „Presby“-Erkrankungen wirken sich allesamt negativ auf die kommunikative und soziale Kompetenz der Senioren aus: Aufgrund der erheblichen kommunikativen Defizite (vor allem durch Presbyphonie und Presbyakusis) sinkt die Lebensqualität älterer Menschen bis hin zu sozialer Isolation mit depressiven Episoden [2,8].

\section{Die Stimme im Alter (Presbyphonie)}

Presbyphonie betrifft die Singstimme (stärkere Beanspruchung des Phonationsapparates) häufiger, früher und deutlicher als die Sprechstimme (geringere Belastung des Phonationsapparates)
$[1,9]$. Dabei sind höhere Singstimmlagen (Sopran, Tenor) aufgrund der stärkeren muskulären Anspannung häufiger und früher betroffen als tiefere Stimmlagen (Alt, Bass) mit geringerer muskulärer Anspannung.

\section{Fachärztliche Untersuchung}

Selbstverständlich ist auch bei altersbedingten Stimmveränderungen eine fachärztliche Untersuchung (Phoniater oder HNOArzt) von Larynx, Stimmgebung und Hörvermögen dringend angezeigt, und zwar

- zum Ausschluss einer Organerkrankung des Kehlkopfes (gutartige oder bösartige Tumoren, Entzündungen, Lähmungen, Ödeme),

- um festzustellen, ob eine medizinische Stimmübungsbehandlung angezeigt ist zur Leistungs- und Qualitätsverbesserung der Stimme,

- um ggf. durch die Verordnung von Hörhilfen eine Verbesserung des audio-phonatorischen Feedback für die eigene Stimme und für fremde Stimmen zu erreichen.

\section{Merkmale der Altersstimme}

Die Altersstimme weist folgende Merkmale auf [1,3,8-14]: Sie wird schwächer, kraftloser, weniger belastbar, rascher ermüdbar, leiser, matt, weniger tragfähig, belegt, behaucht, eingeschränkt modulationsfähig, instabil und brüchig-kippelnd bis zittrig. Der sog. „Alterstremor“ des Larynx mit Stimmzittern $[3,10,11,15]$ wird verursacht einerseits durch eine mangelnde Kontinuität des Atemstromes, andererseits durch eine gestörte zentralnervöse Steuerung und Koordination des Kehlkopfes $[1,9]$. Aufgrund einer Presbyakusis kann die Stimme auch raue, gepresste Anteile aufweisen [7].

\section{Veränderte Singstimme}

Veränderungen der Singstimme im Alter [1,9] zeigen sich u.a. in einer Abnahme der Geläufigkeit: Ältere Sängerinnen und Sänger haben Probleme bei schnellen Tonfolgen und Koloraturen, die Tempi werden schleppender und zäher, schnelles Singen wird insgesamt schwieriger. Weiterhin bestehen Intonationsschwierigkeiten: Die Tontreff-Sicherheit sowie die Koloraturfähigkeit der Singstimme nimmt im Alter ab, es kommt zum Detonieren (Stimmabweichung nach unten). Das Vibrato wird zunehmend größer mit einer Tendenz um Tremolieren. Bei der Singstimmfeld-Messung (Phonetografie) fällt auf, dass Stimmumfang (Ambitus) und Stimmdynamik geringer werden bei häufig fehlender Höhe der Singstimme. Registerübergänge treten stärker hervor, es kommt zu Problemen beim Registerausgleich. Die Brustresonanz nimmt tendenziell ab, die Lautstärkeregulation ist (besonders im Piano) häufig beeinträchtigt. 


\section{Hormonelle Komponente}

Altersstimmprobleme sind hormonell zumindest mitbedingt: Bei Männern kommt es im Rahmen der Andropause zu Testosteronmangel [8], weshalb die mittlere Sprechstimmlage tendenziell höher wird [8-10,13] bis hin zum „Greisendiskant“ (plötzliches Umschlagen der Stimme in die Fistellage). Bei Frauen tritt im Rahmen der Menopause ein zunehmender Östrogenmangel $[8,9]$ mit Überwiegen der Nebennierenrinden-Funktion auf. Dies führt zu einem Absinken der mittleren Sprechstimmlage (sog. „Virilisierung“) $[3,8-10,13,16]$. Derartige hormonbedingte Veränderungen der stimmlichen Tonhöhe/Grundfrequenz treten zwischen dem 45. und 70. Lebensjahr auf. Nach dem Klimakterium nähern sich somit die Grundfrequenzen männlicher und weiblicher Stimmen einander an (die männliche Stimme wird höher, die weibliche Stimme wird tiefer).

\section{Atemantrieb}

Der Atemantrieb wird mit zunehmendem Alter schwächer [8,9], was negativen Einfluss auf die stimmliche Belastbarkeit älterer Menschen hat. Durch die eingeschränkte Lungenfunktion $[1,9,13]$ sinken Tonhaltedauer und Vitalkapazität $[3,8,9,11]$.

\section{Absinken des Kehlkopfs}

Schließlich wird auch die Pharynxmuskulatur schwächer und schlaffer. Diese Hypotonie der Aufhängemuskulatur des Kehlkopfes bewirkt, dass der Larynx im Alter um mehrere Zentimeter absinkt. Das Absinken des Kehlkopfes [13] führt einerseits zu veränderten Resonanzverhältnissen im Ansatzrohr mit Änderung des Stimmklangs, andererseits zu einem Anstieg des Aspirationsrisikos bei Senioren (Presbyphagie).

\section{Der Alterskehlkopf (Presbylarynx)}

Die o.g. stimmlichen Veränderungen beruhen auf morphologischen Altersveränderungen des Kehlkopfes, die seit über 100 Jahren $[17,18]$ immer wieder systematisch untersucht wurden:

\section{Kehlkopfveränderungen}

Die zunehmende Ossifikation der Kehlkopfknorpel (insbesondere Schildknorpel, Ringknorpel und Aryknorpel) [1,8-14] führt zu einer mechanischen Versteifung des Kehlkopfskeletts (Ektolarynx). Die Processus vocales der Aryknorpel treten oftmals prominent hervor $[1,15,19]$. Arthrotische Versteifungen der Crico-Arytenoid-Gelenke $[1,3,8,9,11]$ können zu Bewegungseinschränkungen der Stimmlippen, aber auch zu Schwingungsirregularitäten führen. Arthrotische Versteifungen der Crico-Thyroid-Gelenke können die crico-thyreoidale Approximation und damit die für hohe Stimmlagen erforderliche Spannungserhöhung der Stimmlippen behindern. Arteriosklerotische Durchblutungsstörungen $[1,9,10,13]$ sowie die postklimakterische Atrophie von Schleimhäuten und Schleimdrüsen [1,3, 8-10,13,16] führen häufig im Alter zu einer Laryngitis sicca mit Trockenheitsgefühl im Hals und ständigem Räusperzwang.

\section{Veränderungen der Stimmlippen}

Auch die Stimmlippen selbst weisen charakteristische morphologische Altersveränderungen auf [20-22]: Im M. vocalis kommt es zur Degeneration und Atrophie elastischer Fasern [1,911,14], welche durch Bindegewebsfasern ersetzt werden $[3,8,20,21]$. Die Zahl der Myofibrillen sinkt. Es resultiert eine Vokalisinsuffizienz/Internusschwäche mit Stimmlippenatrophie
$[1,8,9,11-14,16,22]$ und spindelförmig-ovalärem Glottisspalt bei Phonation [1], im angloamerikanischen Schrifttum als „vocal fold bowing“ bekannt $[3,8,10-12,14,15,19]$. Die muskuläre Hypotonie der Stimmlippen [1,9], häufig begleitet von einer fettigen Degeneration elastischer Fasern [13], führt ebenso wie die Laryngitis sicca zu Schwingungsirregularitäten der Stimmlippen $[3,10,15]$. Um den durch die Glottisschlussinsuffizienz $[9,10]$ bedingten Luftverlust während der Phonation zu kompensieren, entwickeln sich häufig supraglottische hyperfunktionelle Pressmechanismen wie Taschenfalteneinsatz oder posterior-anteriore Kontraktionen mit pathologischer Annäherung zwischen Aryknorpeln und Epiglottis [8].

\section{Weitere Veränderungen}

Aber auch die übrigen laryngealen [10], pharyngealen und Atemmuskeln sowie die zugehörigen Bandapparate atrophieren und degenerieren $[1,9]$ mit konsekutiver Hypotonie.

Insgesamt resultiert aus den Veränderungen ein Verlust an Elastizität, Spannkraft und Dehnungsfähigkeit im gesamten Phonations- und Atemapparat [9].

\section{Behandlungsoptionen \\ $\nabla$}

Bei der Stimmrehabilitation im Alter müssen allgemeine und speziell auf die Stimme bezogene Therapieansätze unterschieden werden [9]:

\section{Allgemeine Therapieansätze}

$\mathrm{Zu}$ den allgemeinen Therapieansätzen gehört der Ausschluss oder die Mitbehandlung internistischer und/oder neurologischer Grunderkrankungen im Sinne einer interdisziplinären Basistherapie [9]. So nimmt beispielsweise das Refluxrisiko (und damit das Risiko einer Refluxlaryngitis) im Alter kontinuierlich $\mathrm{zu}[4,23]$. Besonders beachtet werden müssen zerebrovaskuläre Erkrankungen (die zentrale Stimmstörungen bedingen können) und Nebenwirkungen von Medikamenten [1,9,24]. Weitere allgemeine Therapieansätze sind gesunde Lebensweise und naturgemäße Lebensführung, Regelmäßigkeit im Tagesablauf, ausreichend Schlaf, eine vitaminreiche, fettarme und ballaststoffreiche Ernährung sowie die Meidung von Nikotin [9]. Alkohol und Süßigkeiten sollten nur in Maßen genoßen werden, u.a. zur Prävention gastro-ösophago-laryngealer Refluxepisoden.

\section{Spezielle Therapieansätze}

Therapieansätze speziell für die Stimme beinhalten die Einhaltung individueller Sprech- und Gesangspausen zur Vermeidung stimmlicher Überlastung (verlängerte stimmliche und körperliche Erholungszeiten nach Stimmbelastung im Alter! [1,9]). Weiterhin ist auf eine gute Befeuchtung der Atemwege (regelmäßige Flüssigkeitszufuhr, Inhalationen) zu achten, um einer Laryngitis sicca vorzubeugen $[1,9]$. Hormongaben sind sicher nicht die Methode der Wahl bei Altersveränderungen der Stimme, sie sollten nur bei stärkeren klimakterischen Beschwerden und auch dann nur unter regelmäßiger fachärztlicher Kontrolle, nach strenger Indikationsstellung, möglichst gering dosiert und nur über einen begrenzten Zeitraum erfolgen [9]. Zudem müssen lebensgefährliche Nebenwirkungen (Mamma-Karzinom und Herzinfarkt bei Frauen bei regelmäßiger Östrogeneinnahme, Prostatakarzinom 
bei Männern bei regelmäßiger Testosteroneinnahme) berücksichtigt und mit den Betroffenen besprochen werden.

Aufgrund der muskulären (insbesondere glottischen) Atrophie und Hypotonie kommen in der Stimmtherapie vorwiegend tonisierende Übungen zur Verbesserung des phonatorischen Glottisschlusses (im angloamerikanischen Schrifttum als „glottal attack exercises“ bekannt [8]) in Betracht. Supraglottische hyperfunktionelle Pressmechanismen sollten durch detonisierende Entspannungsübungen abgebaut werden [8]. Bei Sängern sollte das Liedgut altersadaptiert werden (z.B. weniger dramatische und mehr lyrische Partien, evtl. Eingruppierung in tiefere Stimmlagen), eine gesangspädagogische Mitbetreuung ist oftmals hilfreich $[9,25]$. Phonochirurgisch („operative Klangpflege“/ „Stimmtuning“) kommt die paraglottische (zwischen Schildknorpel und Vokalismuskel) Injektion von Kollagen, Hyaluronsäure oder körpereigenem Fett in Betracht, um bei einer ausgeprägten Glottisschlussinsuffizienz mit Vokalismuskel-Atrophie den phonatorischen Glottisschluss zu verbessern und damit die Stimme zu kräftigen $[1,8,11,12]$. Die Patienten müssen jedoch darüber informiert werden, dass diese Substanzen durch körpereigene Abbauprozesse in durchschnittlich 3-6 Monaten resorbiert werden. Um einen dauerhaft guten phonatorischen Glottisschluss zu erreichen, müssen die Injektionen somit regelmäßig wiederholt werden.

\section{Therapeutische Effekte des Singens im Alter}

$\nabla$

\section{Psychosoziale Effekte}

Gesangspädagogische Betreuung, z.B. mit dem Anti-AgingStimmtrainings-Programm nach Bengtson-Opitz [25], fördert nicht nur die stimmliche Belastbarkeit. Singen im Alter hat darüber hinaus auch positive psychosoziale Effekte (Verbesserung der sozialen Teilhabe, Reduktion von Angst und Depressionen, Steigerung des körperlichen und seelischen Wohlbefindens und der Stimmungslage, Förderung gemeinschaftlichen Empfindens und Erlebens, allgemein Steigerung der Lebensqualität). Diese salutogenetischen Effekte des Singens im Alter wurden in etlichen Studien wiederholt nachgewiesen [26-29]. Chorsingen könnte sogar die Lebenserwartung verlängern [30].

\section{Immunologische Effekte}

Auch in experimentellen laborchemischen Studien konnten positive Gesundheitseffekte durch Chorsingen und Musizieren nachgewiesen werden: So steigert Singen statistisch signifikant die Produktion von Immunglobulin A (IgA) im Speichel und schützt damit die oberen Atemwege vor Infektionen [31-33]. Singen stimuliert also das Immunsystem und stärkt die Abwehrkräfte, was insbesondere für Senioren elementar wichtig ist. In den gleichen Studien [31-33] konnten mit psychometrischen Rating-Skalen auch signifikante Verbesserungen des seelischen Wohlbefindens und der Stimmungslage beim Chorsingen gemessen werden.

Singen im Alter hat demnach positive psychosoziale und immunologische Auswirkungen.

\section{Fazit}

Etwa $20 \%$ der über 60-Jährigen leiden unter Stimmproblemen. Diese Stimmveränderungen im Alter (Presbyphonie) gehen einher mit morphologischen Alterungsprozessen im Kehlkopf (Presbylarynx). Therapeutische Optionen beinhalten einerseits die Behandlung internistischer und neurologischer Grunderkrankungen, andererseits einen gesunden Lebensstil zum Funktionserhalt des Atem- und Stimmapparates. Stimmtherapeutisch wären zu nennen: Tonisierungsübungen auf Glottisebene, Abbau supraglottischer Pressmechanismen, ggf. phonochirurgische paraglottische Injektionen zur Verbesserung des phonatorischen Glottisschlusses, sowie stimmhygienische Maßnahmen (z.B. regelmäßige Befeuchtung der Atemwege). Singen im Alter hat sowohl psychosozial als auch immunologisch positive Effekte.

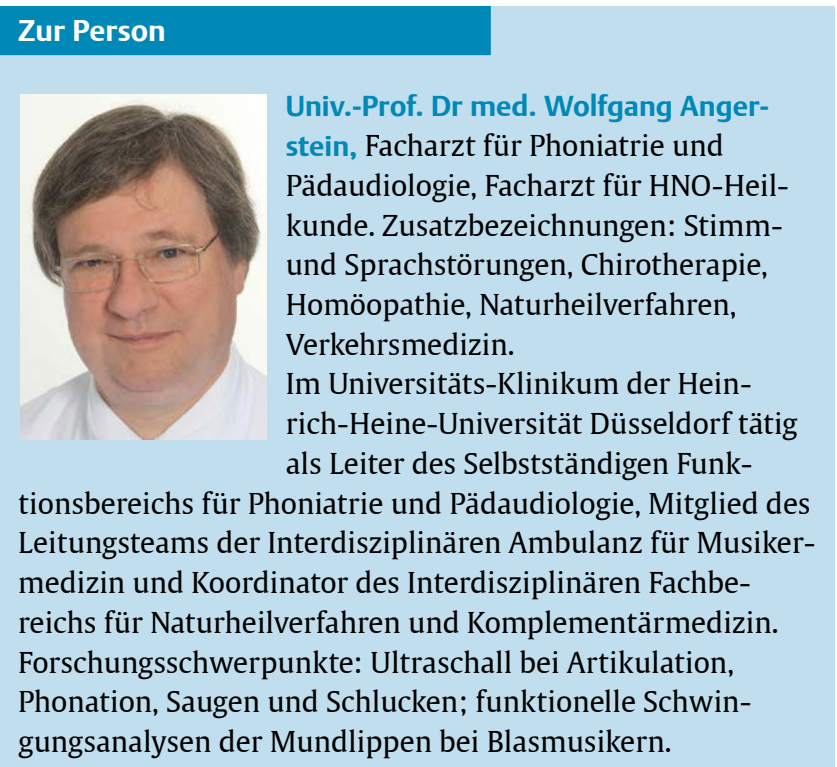

Interessenkonflikt: Der Autor gibt an, dass kein Interessenkonflikt besteht.

\section{Literatur}

1 Am Zehnhoff-Dinnesen A, Angerstein W, Deuster D. Communication disorders in the elderly. Audiolog Med 2010; 8: 184-190

2 Cohen SM, Turley R. Coprevalence and impact of dysphonia and hearing loss in the elderly. Laryngoscope 2009; 119: 1870-1873

3 Kendall K. Presbyphonia: a review. Curr Opinion Otolaryngol Head Neck Surg 2007; 15: 137-140

4 Roy N, Stemple J, Merrill RM et al. Epidemiology of voice disorders in the elderly: preliminary findings. Laryngoscope 2007; 117: 628-633

5 Turley $R$, Cohen $S$. Impact of voice and swallowing problems in the elderly. Otolaryngol Head Neck Surg 2009; 140: 33-36

6 Tesch-Römer C. Schwerhörigkeit im Alter. Heidelberg: Median; 2001

7 Weatherley CC, Worrall LE, Hickson LM. The effect of hearing impairment on the vocal characteristics of older people. Folia Phoniatr Logop 1997; 49: 53-62

8 Hagen P, Lyons GD, Nuss DW. Dysphonia in the elderly: diagnosis and management of age-related voice changes. South Med J 1996; 89: 204-207

9 Angerstein W. Das Altern der Stimme - Dem "Schicksal in den Rachen greifen". HNO-Nachrichten 2008; 38: 34-38

10 Ramig LO, Gray S, Baker K et al. The aging voice: a review, treatment data and familial and genetic perspectives. Folia Phoniatr Logop 2001; 53: $252-265$ 
11 Sataloff RT. Vocal aging. Curr Opinion Otolaryngol Head Neck Surg 1998; 6: 421-428

12 Sinard RJ. The aging voice: how to differentiate disease from normal changes. Geriatrics 1998; 53: 76-79

13 Sonies BC, Stone M, Shawker T. Speech and swallowing in the elderly. Gerodontology 1984; 3: 115-123

14 Verdonck-de Leeuw IM, Mahieu HF. Vocal aging and the impact on daily life: a longitudinal study. J Voice 2003; 18: 193-202

15 Pontes $P$, Yamasaki R, Behlau M. Morphological and functional aspects of the senile larynx. Folia Phoniatr Logop 2006; 58: 151-158

16 D'haeseleer E, Depypere H, Claeys S et al. The menopause and the female larynx, clinical aspects and therapeutic options: a literature review. Maturitas 2009; 64: 27-32

17 Imhofer $R$. Ueber das elastische Gewebe im Stimmbande alter Individuen. Cbl Allg Path Patholog Anat 1914; 25: 337-339

18 Kofler K. Der Alterskehlkopf. Wiener Med Wschr 1931; 48: 1583-1585

19 Pontes P, Brasolotto A, Behlau M. Glottic characteristics and voice complaint in the elderly. J Voice 2005; 19: 84-94

20 Hirano M, Kurita S, Sakaguchi S. Ageing of the vibratory tissue of human vocal folds. Acta Otolaryngol (Stockh) 1989; 107: 429-433

21 Sato K, Hirano M, Nakashima T. Age-related changes of collagenous fibers in the human vocal fold mucosa. Ann Otol Rhinol Laryngol 2002; 111: $15-20$

22 Takano S, Kimura M, Nito $T$ et al. Clinical analysis of presbylarynx Vocal fold atrophy in elderly individuals. Auris Nasus Larynx 2010; 37: 461-464

23 Lee J, Anggiansah A, Anggiansah $R$ et al. Effects of age on the gastroesophageal junction, esophageal motility, and reflux disease. Clin Gastroenterol Hepatol 2007; 5: 1392-1398

24 Martin FG. Tutorial: drugs and vocal function. J Voice 1988; 2: 338-344
25 Bengtson-Opitz E. Anti-Aging für die Stimme. Ein Trainingsprogramm für den strapazierten Stimmapparat. Sprache-Stimme-Gehör 2007; 31: $60-65$

26 Berg EE, Hapner E, Klein A et al. Voice therapy improves quality of life in age related dysphonia: a case-control study. J Voice 2008; 22: 70-74

27 Clift S, Hancox G. The significance of choral singing for sustaining psychological well-being: findings from a survey of choristers in England, Australia and Germany. Music Performance Res 2010; 3 (special issue "Music and Health"): 79-96

28 Cohen GD, Perlstein S, Chapline J et al. The impact of professionally conducted cultural programs on the physical health, mental health, and social functioning of older adults. Gerontologist 2006; 42: 20-48

29 Houston DM, McKee KJ, Caroll L et al. Using humour to promote psychological wellbeing in residential homes for older people. Aging and Mental Health 1998; 2: 328-332

30 Bygren LO, Konlaan BB, Johansson SE. Attendance at cultural events, reading books or periodicals, and making music or singing in a choir as determinants for survival: Swedish interview survey of living conditions. BMJ 1996; 313: 1577-1580

31 Kreutz G, Bongard S, Rohrmann S et al. Effects of choir singing or listening on secretory immunoglobulin A, cortisol, and emotional state. J Behav Med 2004; 27: 623-635

32 Beck RJ, Cesario TC, Yousefi A et al. Choral singing, performance perception, and immune system changes in salivary immunoglobulin A and cortisol. Music Perception 2000; 18: 87-106

33 McCraty R, Atkinson M, Rein G et al. Music enhances the effect of positive emotional states on salivary IgA. Stress Med 1996; 12: 167-175 